\title{
A projection for psychiatry in the post-COVID-19 era: potential trends, challenges, and directions
}

\author{
Halide Bilge Türközer (iD) ${ }^{1}$ Dost Öngür ${ }^{2,3}$
}

Received: 28 April 2020 / Revised: 2 July 2020 / Accepted: 7 July 2020 / Published online: 17 July 2020

(c) Springer Nature Limited 2020

\begin{abstract}
The COVID-19 pandemic has transformed the face of psychiatry over a very short time period. Given the detrimental impact of the pandemic on mental health and the economy, more difficult days are ahead for psychiatry. The rising public health burden of mental illnesses will inevitably exceed the capacity of psychiatric services in the United States and worldwide. The pandemic has also profoundly affected psychiatric research due to safety concerns and containment efforts. Intermediate and long-term ramifications may even be more serious. In addition to the effects of the economic downturn on available research funding, existing research tools and protocols may not meet the emerging needs in the post-COVID-19 era. This paper discusses potential trends and challenges that psychiatric practice and research may encounter in this period from the viewpoint of workers in the field. We outline some measures that clinicians and researchers can implement to adapt to the emerging changes in psychiatry and to mitigate the forthcoming effects of the crisis.
\end{abstract}

The COVID-19 pandemic has created a sense of danger, uncertainty, and loss of control in populations worldwide, placing mental health discussions high on the public's agenda. Disasters traumatize societies, typically in a timelimited event with destructive outcomes that hit one community and require others to help. The pandemic is unusual because the world faces a danger with an unknown end date. Communities that support each other in normal times are now competing for scarce resources to cope with their own crises. The impact of this crisis on individuals and societies is compounded by the experience of facing danger without help. If the pandemic lasts for an extended period, as projected by some models [1,2], psychiatric practice and the place of psychiatry in medicine are likely to undergo lasting changes. Here, we will (i) identify potential trends and challenges that psychiatric practice and research may

Halide Bilge Türközer

bilge.turkozer@gmail.com

1 Department of Psychiatry, The University of Texas Southwestern Medical Center, Dallas, TX, USA

2 Psychotic Disorders Division, McLean Hospital, Belmont, MA, USA

3 Department of Psychiatry, Harvard Medical School, Boston, MA, USA encounter during this period, (ii) will suggest concrete measures that clinicians and researchers can take to mitigate the effects of this crisis at individual and institutional levels.

\section{A projection for psychiatric clinical practice}

In this acute phase of the crisis, psychiatrists face unique challenges including caring for patients with serious mental illnesses and COVID-19 infections [3-5], preventing spread in acute psychiatry units, and providing emergency mental health services. In the outpatient world, the practice of psychiatry has transformed over just a couple of weeks [6]. Telepsychiatry, which used to account for a small portion of psychiatric services [7], has become the new norm. A similar transition to telemedicine is also underway for consultation-liaison services [8] and even for inpatient psychiatry units.

However, more challenging days are on the horizon for psychiatry. The current climate is a perfect incubator for rising public health burden due to psychiatric disorders $[9,10]$. The pandemic has a negative impact on mental health while stretching psychiatric services to the limit and reducing our ability to respond due to economic downturn. Although the long-term ramifications of the economic downturn are currently unclear and the downturn will affect different sectors of society differently, the pandemic 
threatens a prolonged economic crisis due to the costs associated with social distancing measures lasting for months $[11,12]$. An economic recession, by itself, brings the risk of an increase in psychiatric disorders [13], homelessness [14], and suicide rates [15]. It is also likely that the rate of substance use disorders will surge [16], adding to the major opioid crisis in the United States and worldwide [17]. This cascade of events makes it highly possible that the demand for mental health services will exceed the existing capacity of the system. We must prepare now to meet this challenge.

Uncomplicated depression, anxiety, substance use disorders, and sleep disorders are among the most common psychiatric disorders worldwide. A large proportion of patients with these disorders can be effectively treated in primary healthcare settings. These disorders are also the leading conditions that will be exacerbated by the pandemic [10]. Perhaps the most effective short-term solution to the looming crisis is to improve the knowledge and confidence of primary care physicians in treating these prevalent psychiatric disorders. The effectiveness of this approach, the primary care first (PCP-First) model, has been well studied and supported by randomized controlled trials over the last two decades [18-20]. The Collaborative Care Model, another primary care-based model that integrates physical and behavioral health services, is also clinically and costeffective for the treatment of various psychiatric disorders [21-24]. There is an urgent need for policies that promote the implementation of these models in nonpsychiatric care settings broadly across the country. The changes in psychiatric practice following the pandemic may also stimulate improvements in these models. For example, high attrition rates and inadequate clinician/staff time are some of the major limitations to the PCP-First model $[20,25]$. The undergoing transformation in telemedicine may accelerate the development of smart-phone apps or web-based surveys that would allow remote (and wider) screening and help patients interact with clinicians before and between scheduled visits. These developments may help overcome the major obstacles to the widespread adoption of these models $[20,25]$.

Another potential impact of this climate would be on the place of psychiatry within medicine. The need for psychiatric services and devastating outcomes in the absence of services will become more visible following the crisis. It will become more clear that a group of medical problems that affect one-fourth of the population [26] cannot be solely treated by specialists. Clinicians will more frequently see mental health problems affect their patients' overall health. In the absence of access to specialty services, primary care and other physicians will need to become even more proactive in treating their patients' uncomplicated psychiatric problems. This necessity may also increase the weight of psychiatric education in medical education curricula. The lack of adequate mental health services will increase the interest in preventive interventions in psychiatry.

In the short-term, there are some steps that outpatient psychiatrists and clinical entities can take to prepare for a potential surge in the demand for mental health services.

\section{Measures that outpatient clinicians can take}

- Identify patients under your care who are stable and can be transferred to the care of their PCP's if needed in case of an overwhelming clinical load.

- Initiate conversations with those patients and their PCP's on a potential transfer of care if required. Provide assurance that you will continue to be available when needed.

- Reach out to PCP's in your local community and discuss the potential effects of the pandemic on psychiatric services. Provide them with resources on the diagnosis and treatment of uncomplicated mental illnesses. Offer your help as a consultant when possible.

- Identify patients who are at risk for relapse and reach out to them proactively.

- Revise the admission process in your clinic. Prepare an "emergency triage plan" to put in place if clinical load exceeds your clinic's capacity. Provide referral guidelines to physicians that frequently refer patients to your clinic. Encourage them to try first-step treatments before referrals.

- When possible, adapt a consultation-based care model where the primary care physician continues to provide mental healthcare after a consultation visit with the psychiatrist.

- Prepare a strategy for your clinic to adopt digital health technology into your practice.

\section{Measures that clinical entities can take}

- Implement collaborative care models in your institution to integrate mental health and primary care services.

- Prepare internal trainings on the diagnosis and treatment of common mental illnesses for nonpsychiatric clinicians.

- Initiate universal screenings for depression, substance use, and anxiety disorders in outpatient clinics.

- Prepare internal referral guidelines to warrant an initial trial of first-step treatments in primary care settings before referrals.

- Organize virtual school and public outreaches to provide psychoeducation on the effects of the pandemic on mental health and when to seek help. 
These preparations are critical to collectively carry the public health burden of greater psychiatric morbidity in societies. Psychiatry will continue to provide critical specialty services, but it cannot meet this challenge alone.

These unprecedented circumstances may also lead to psychiatric presentations that have not been commonly seen before. The COVID-19 pandemic introduces a new type of mass trauma [27]. It is unique in the ways that it significantly affects every aspect of individuals' daily lives, it is global in scope, and individuals witness the imminent life-threatening danger through electronic media and television in most cases. Could the exposure to news reporting hundreds of people dying every day in your community for months be considered a traumatic event? Potentially, yes and at a minimum, there will be significant experiences with adjustment disorder in the population. Thus, significant traumatic responses may fall between the diagnostic cracks [27]. Ultimately, it is difficult at the current time to get a true estimation of the scope of traumatic responses seen in the population in response to the pandemic. COVID-19 related delusions that affect functionality or social anxiety due to the fear of spread are some other presentations that are already emerging. Given the scope of the crisis worldwide, these presentations may become common. That would create a need for new diagnostic categories or specifiers.

The COVID-19 pandemic put healthcare professionals and first responders under extreme physical and psychological stress across the world. Institutions and mental health professionals have responded to this crisis by implementing psychological intervention services that offer mental health support hotlines and guidance on how to cope with stress and develop resilience [28-30]. Although these services have helped many frontline workers, there are significant limitations to these interventions. Commonly highlighted coping strategies such as behavioral activation and mindfulness practices [28] are habits that require time and effort to develop. Individuals who have these tools in their toolbox benefit from them at the time of a crisis. However, it is less likely to succeed in developing new habits in times of crisis. Secondly, participating in these intervention programs also requires time and effort. Chen et al. recently published their experience in implementing psychological intervention services in a tertiary hospital in China during the COVID-19 outbreak [29]. A major obstacle that they encountered was the reluctance of staff to participate in these interventions, stating that they "did not need a psychologist, but needed more rest without interruption". To overcome this barrier, the hospital provided the staff with an isolated place to rest, guaranteed food and daily living supplies, and made psychological counselors available onsite to provide support during break times [29]. Avoidance, a core symptom of trauma, may also prevent healthcare workers from reaching out for help and using these services. Individuals who are at lower levels of stress and less burnt out may be more likely to participate in these supportive services [31]. Team leaders should actively reach out to team members who are repeatedly not available to participate in support meetings [30].

The COVID-19 pandemic magnified the inadequacy of the healthcare system in attending the needs of healthcare professionals and preparing them psychologically and physically for their crucial roles. This is a time that we need to go beyond "Wellness Committees". Sports medicine and space medicine have been utilizing evidence-based approaches to prepare athletes and astronauts for highly demanding, high-stress, and unpredictable duties. Similar to their approach, there is a need for an interdisciplinary field that focuses on improving mental and physical health in healthcare professionals and first responders, in addition to providing early support at the time of a crisis and aftercare. Preventive interventions, such as teaching behavioral strategies for coping with stress, should be embedded in their professional training in a way that they can form these habits during their training (e.g., weekly practices as a part of the curriculum, instead of a single lecture on this topic). Monitoring healthcare workers for mental health illnesses and providing early support will remain crucial in the aftermath of the pandemic. The World Trade Center (WTC) Health Program sets an example that provides longitudinal physical and mental healthcare to first responders, in addition to collecting epidemiological data on the long-term health consequences of the disaster [32]. The WTC Health Program may help inform future efforts to provide continuing support to healthcare workers after the pandemic. If we fail to take on this urgent task to better support the physical and mental wellbeing of healthcare workers, the workforce shortage in healthcare will continue to grow at an increased pace after the pandemic.

\section{A projection for psychiatric research practice}

The rapid changes currently taking place in response to the COVID-19 pandemic will undoubtedly affect mental health research. Recent publications outlined research priorities to understand the effects of the COVID infection and the pandemic on mental health from psychological, social, and neuroscientific perspectives [33, 34]. Here, we will discuss the effects of the pandemic on practical and logistic aspects of psychiatric research. We will explore potential trends and challenges in clinical research, research practices, available funding, and scientific publications. We will seek answers to a less-explored question: how will the life of a psychiatric researcher change in the postCOVID era? 


\section{Special considerations for clinical research domains}

The pandemic brought both challenges and opportunities for clinical researchers. Plenty of clinical questions have been raised and funding opportunities are now available to investigate these questions [35, 36]. However, clinical research is not immune to the emerging challenges that psychiatric practice encounters [37]. Currently, the recruitment of participants for clinical trials is largely on hold to prevent the spread of the COVID-19 infection. We do not know when-and if-researchers will be able to resume their regular operations.

There are many reasons why researchers should prepare for changes in their research operations. First, even though social distancing practices may ease in the near future, the fear of contamination in medical settings will likely last longer. Due to the increased sense of danger, individuals will be less willing to come into the hospitals for clinical trials. Particularly trials that do not offer treatment interventions will have greater difficulty recruiting patients. The recruitment of healthy individuals may become even more challenging. To mitigate this problem clinical research will need to adopt digital technologies into research designs. Even if clinical trials are able to implement digital technologies, connectivity may become a logistic barrier for many people with psychiatric problems. Research in some fields, such as psychotic disorders, may be more seriously impacted by these barriers due to more severe socioeconomic disadvantages seen in those populations. The difficulty in recruiting participants may also require researchers to increase the amount of monetary compensation for participation in clinical trials-as approved by Institutional Review Boards (IRB).

Second, existing research tools may not meet the emerging needs of clinical researchers in the post-COVID-19 era. Adaptation of digital technologies into clinical research will require compatible and validated research tools for digital settings. There are a number of potential challenges to the validity of online clinical assessments, largely due to the lack of control in the testing situation and environmental factors influencing responses. Studies comparing online and traditional versions of psychological tests demonstrate that online tests can have acceptable psychometric properties, but may show significant differences in factor structures [38]. For this reason, there is a need for the validation of commonly used clinical assessments for virtual settings. Similarly, cognitive assessment tools may need to be validated and re-normed for virtual visits. Online cognitive assessments may be increasingly utilized in this period. Although there are online cognitive assessments that have been validated in different patient populations [39-42], studies on unsupervised online assessments are limited [43]. The increasing need for remote assessments and IRB's rapid responses to these requests may also accelerate the developments in digital app-based research.

Third, the pandemic poses an unaccounted confounding factor for randomized clinical trials, which employ rigorous controls to reduce variation and bias. Stress reactions seen in response to the pandemic may affect the response to clinical interventions and skew clinical outcomes. Will participants who have acute decompensations after the pandemic be excluded from data analyses? What about participants who are infected with COVID? Researchers will need to develop standardized protocols to address these questions. Additional clinical measures that were not considered relevant before may now be needed to account for confounding effects that the pandemic introduced. The development of standardized clinical tools that measure the impact of the pandemic on different mental health domains may help researchers develop standard methods to account for these factors across different trials. Attrition rates may also increase in this period. Dropouts may be associated with specific demographic or clinical features that are distinctively affected by the pandemic, which would introduce additional bias [44]. Researchers may need to gather more detailed information on the reasons for protocol nonadherence following the pandemic to be able to classify their missing data, which would guide their data analysis strategy.

Neuroimaging, and biomarker research in general, may take a harder hit in this period. In addition to the recruitment difficulties outlined above, there is the issue of the safety of the research personnel and the environment. In addition to adopting higher-level personal protection precautions, testing participants and research personnel for SARS-CoV-19 may become a routine practice before procedures that require long in-person visits and patient contact, such as EEG or imaging. Pre-procedure COVID testing has already become a routine practice before outpatient surgeries and ECT in some institutions. However, limited financial resources could be a barrier to its implementation by research programs. Imaging facilities may need to make arrangements on MRI scanners and scanning environments to be able to scan patients with COVID-19 infections, which is essential to enhance our knowledge of the clinical characteristics of the infection. Psychiatric genetics, which has already adopted remote data gathering into their practices [45], may be less impacted in this period.

\section{Potential ramifications for research funding}

The urgent need for research on various aspects of the COVID-19 pandemic will shift funding resources to this new field. Given the economic downturn that the pandemic has caused, which is expected to have long-term effects, available funding will become even more scarce. The 
allocation of already limited resources will likely hinder the development of other fields in psychiatry. Researchers will need to modify their short and long-term strategies to be able to obtain funding. These strategies may include integrating aspects of COVID-related research into their planned projects or potentially shifting their research areas. The use of high-tech and expensive equipment (such as MRI scans) will likely decrease as researchers will have higher chances of being funded for projects with lower budgets. They may also take the strategy to use existing databases while adopting more complex statistical methods. This climate would further promote industry-funded research which would take a toll on the already-struggling independent research.

\section{Considerations for academic publishing and research culture}

The shift in psychiatric research will undoubtedly affect scientific publishing in psychiatry. The influx of COVIDrelated submissions has already overwhelmed major journals. Due to the urgent need and high demand for these articles, journals prioritize publishing these papers over research on other topics, even if they have a lower level of scientific evidence or promise of progress. This may also result in the blurring of traditional lines of the scopes of journals. Journals may need to develop strategies to continue to publish high-quality research within their traditional scope. As researchers may prefer to hold off on submitting new papers in this period, the use of preprint servers may become more common. Researchers may need to be increasingly careful about predatory open-access journals in this period.

Even though the pandemic has impeded global connectedness, the academic discourse has become more international in response to the global need for shared experience and knowledge. Difficulties in securing funding and recruitment outlined above will likely foster national and international collaborations. These joint efforts would allow more efficient use of limited funding resources. As a result, these developments may transform the culture-bound nature of research and academic publishing in the near future.

\section{Conclusions}

The COVID-19 pandemic is changing the face of psychiatry permanently. Upcoming challenges will be significant. In order to serve the needs of our patients and of society, psychiatrists and researchers will need to remain nimble, forward-thinking, and ready to adapt to new situations.
Acknowledgements HBT is supported by the NIMH (R25MH101078, PI: Madhukar H. Trivedi, MD). DO is supported by the NIMH (K24MH104449).

\section{Compliance with ethical standards}

Conflict of interest The authors declare that they have no conflict of interest.

Publisher's note Springer Nature remains neutral with regard to jurisdictional claims in published maps and institutional affiliations.

\section{References}

1. Murray CJL. Forecasting COVID-19 impact on hospital bed-days, ICU-days, ventilator-days and deaths by US state in the next 4 months. MedRxiv. 2020. https://doi.org/10.1101/2020.03.27. 20043752.

2. COVID-19: what's new for April 21. The Institute for Health Metrics and Evaluation. 2020. http://www.healthdata.org/sites/ default/files/files/Projects/COVID/Estimation_update_042120. pdf.

3. Druss BG. Addressing the COVID-19 pandemic in populations with serious mental illness. JAMA Psychiatry. 2020. https://doi. org/10.1001/jamapsychiatry.2020.0894.

4. Xiang YT, Zhao YJ, Liu ZH, Li XH, Zhao N, Cheung T, et al. The COVID-19 outbreak and psychiatric hospitals in China: managing challenges through mental health service reform. Int J Biol Sci. 2020;16:1741-4.

5. Li W, Yang Y, Liu ZH, Zhao YJ, Zhang Q, Zhang L, et al. Progression of mental health services during the COVID-19 outbreak in China. Int J Biol Sci. 2020;16:1732-8.

6. Wright JH, Caudill R. Remote treatment delivery in response to the COVID-19 pandemic. Psychother Psychosom. 2020;89:1-3.

7. Spivak S, Spivak A, Cullen B, Meuchel J, Johnston D, Chernow $\mathrm{R}$, et al. Telepsychiatry use in U.S. mental health facilities, 2010-2017. Psychiatr Serv. 2020;71:121-7.

8. Greenhalgh T, Wherton J, Shaw S, Morrison C. Video consultations for covid-19. BMJ. 2020;368:m998.

9. Galea S, Merchant RM, Lurie N. The mental health consequences of COVID-19 and physical distancing: the need for prevention and early intervention. JAMA Intern Med. 2020;180:817-8.

10. Pfefferbaum B, North CS. Mental health and the Covid-19 pandemic. N Engl J Med. 2020. https://doi.org/10.1056/ NEJMp2008017.

11. Atkeson A. What will be the economic impact of COVID-19 in the US? Rough estimates of disease scenarios. NBER Work Pap Ser. 2020:w26867. https://doi.org/10.3386/w26867.

12. Arellano C, Bai Y, Mihalache GP. Deadly debt crises: COVID-19 in emerging markets. NBER Work Pap Ser. 2020:w27275. https:// doi.org/10.3386/w27275.

13. Katikireddi SV, Niedzwiedz CL, Popham F. Trends in population mental health before and after the 2008 recession: a repeat crosssectional analysis of the 1991-2010 health surveys of England. BMJ Open. 2012;2:e001790.

14. Bainbridge J, Carrizales T. Global homelessness in a postrecession world. J Public Manag Soc Policy. 2017;24:6.

15. Coope C, Gunnell D, Hollingworth W, Hawton K, Kapur N, Fearn V, et al. Suicide and the 2008 economic recession: who is most at risk? Trends in suicide rates in England and Wales 2001-2011. Soc Sci Med. 2014;117:76-85. 
16. Clay JM, Parker MO. Alcohol use and misuse during the COVID19 pandemic: a potential public health crisis? Lancet Public Health. 2020;5:e259.

17. Alexander GC, Stoller KB, Haffajee RL, Saloner B. An epidemic in the midst of a pandemic: opioid use disorder and COVID-19. Ann Intern Med. 2020. https://doi.org/10.7326/M20-1141.

18. Schulberg HC, Katon W, Simon GE, Rush AJ. Treating major depression in primary care practice: an update of the agency for health care policy and research practice guidelines. Arch Gen Psychiatry. 1998;55:1121-7.

19. Trivedi MH, Rush AJ, Wisniewski SR, Nierenberg AA, Warden D, Ritz L, et al. Evaluation of outcomes with citalopram for depression using measurement-based care in STAR*D: implications for clinical practice. Am J Psychiatry. 2006;163:28-40.

20. Trivedi MH, Jha MK, Kahalnik F, Pipes R, Levinson S, Lawson T, et al. Vitalsign6: a primary care first (PCP-first) model for universal screening and measurement-based care for depression. Pharmaceuticals. 2019;12:71.

21. Richards DA, Hill JJ, Gask L, Lovell K, Chew-Graham C, Bower $\mathrm{P}$, et al. Clinical effectiveness of collaborative care for depression in UK primary care (CADET): Cluster randomised controlled trial. BMJ. 2013;347:f4913.

22. Gilbody S, Bower P, Fletcher J, Richards D, Sutton AJ. Collaborative care for depression: a cumulative meta-analysis and review of longer-term outcomes. Arch Intern Med. 2006; 166:2314-21.

23. Watkins KE, Ober AJ, Lamp K, Lind M, Setodji C, Osilla KC, et al. Collaborative care for opioid and alcohol use disorders in primary care: the SUMMIT randomized clinical trial. JAMA Intern Med. 2017;177:1480-8.

24. Unützer J, Harbin H, Schoenbaum M, Druss BG. The collaborative care model: an approach for integrating physical and mental health care in Medicaid health homes. Health Home Information Resource Center. 2013;1-13. http://www.chcs.org/media/HH_ IRC_Collaborative_Care_Model_052113_2.pdf.

25. Jha MK, Grannemann BD, Trombello JM, Clark EW, Eidelman SL, Lawson T, et al. A structured approach to detecting and treating depression in primary care: VitalSign6 project. Ann Fam Med. 2019;17:326-35.

26. The World Health Report 2001: Mental health: new understanding, new hope. 2001. https://www.who.int/whr/2001/en/w hr01_en.pdf?ua=1. Accessed 22 Apr 2020.

27. Horesh D, Brown AD. Covid-19 response: traumatic stress in the age of Covid-19: a call to close critical gaps and adapt to new realities. Psychol Trauma. 2020;12:331-5.

28. Polizzi C, Lynn SJ, Perry A. Stress and Coping in the Time of COVID-19: pathways to resilience and recovery. Clin Neuropsychiatry. 2020;17:59-62.

29. Chen Q, Liang M, Li Y, Guo J, Fei D, Wang L, et al. Mental health care for medical staff in China during the COVID-19 outbreak. Lancet Psychiatry. 2020;7:e15-6.

30. Greenberg N, Docherty M, Gnanapragasam S, Wessely S. Managing mental health challenges faced by healthcare workers during covid-19 pandemic. BMJ. 2020;368:m1211.

31. Ledikwe JH, Kleinman NJ, Mpho M, Mothibedi H, Mawandia S, Semo BW, et al. Associations between healthcare worker participation in workplace wellness activities and job satisfaction, occupational stress and burnout: a cross-sectional study in Botswana. BMJ Open. 2018;8:e018492.

32. Dasaro CR, Holden WL, Berman KD, Crane MA, Kaplan JR, Lucchini RG, et al. Cohort profile: world trade center health program general responder cohort. Int J Epidemiol. 2017;46:e9.

33. Holmes EA, O'Connor RC, Perry VH, Tracey I, Wessely S, Arseneault L, et al. Multidisciplinary research priorities for the COVID-19 pandemic: a call for action for mental health science. Lancet Psychiatry. 2020;7:547-60.

34. Nicol GE, Karp JF, Reiersen AM, Zorumski CF, Lenze EJ. What were you before the war?' Repurposing psychiatry during the COVID-19 pandemic. J Clin Psychiatry. 2020; $81: 20$ com 13373 .

35. NIH. Notice of special interest (NOSI): NIA availability of administrative supplements and revision supplements on coronavirus disease 2019 (COVID-19). 2020. https://grants.nih.gov/ grants/guide/notice-files/NOT-AG-20-022.html. Accessed 27 Apr 2020.

36. National Science Foundation. Dear colleague letter on the coronavirus disease 2019 (COVID-19). 2020. https://www.nsf.gov/ pubs/2020/nsf20052/nsf20052.jsp. Accessed 27 April 2020.

37. National Institute of Health. Guidance for NIH-funded clinical trials and human subjects studies affected by COVID-19. 2020. https://grants.nih.gov/grants/guide/notice-files/NOT-OD20-087.html. Accessed 27 April 2020.

38. Buchanan T. Online assessment: desirable or dangerous? Prof Psychol Res Pract. 2002;33:148-54.

39. Domen AC, Weijer SCF, Jaspers MW, Denys D, Nieman DH. The validation of a new online cognitive assessment tool: the MyCognition quotient. Int J Methods Psychiatr Res. 2019;28: e1775.

40. Silverstein SM, Berten S, Olson P, Paul R, Williams LM, Cooper $\mathrm{N}$, et al. Development and validation of a World-Wide-Web-based neurocognitive assessment battery: WebNeuro. Behav Res Methods. 2007;39:940-9.

41. Lees J, Applegate E, Emsley R, Lewis S, Michalopoulou P, Collier T, et al. Calibration and cross-validation of MCCB and CogState in schizophrenia. Psychopharmacology. 2015;232: 3873-82.

42. Maruff P, Thomas E, Cysique L, Brew B, Collie A, Snyder P, et al. Validity of the CogState brief battery: Relationship to standardized tests and sensitivity to cognitive impairment in mild traumatic brain injury, schizophrenia, and AIDS dementia complex. Arch Clin Neuropsychol. 2009;24:165-78.

43. Mackin RS, Insel PS, Truran D, Finley S, Flenniken D, Nosheny $\mathrm{R}$, et al. Unsupervised online neuropsychological test performance for individuals with mild cognitive impairment and dementia: results from the Brain Health Registry. Alzheimer's Dement Diagn Assess Dis Monit. 2018;10:573-82.

44. Leon AC, Mallinckrodt CH, Chuang-Stein C, Archibald DG, Archer GE, Chartier K. Attrition in randomized controlled clinical trials: methodological issues in psychopharmacology. Biol Psychiatry. 2006;59:1001-5.

45. Geschwind DH, Flint J. Genetics and genomics of psychiatric disease. Science. 2015;349:1489-94. 